\title{
VIOLENCIA DE GÉNERO EN ESTUDIANTES DE LICENCIATURA DEL IPN
}

\section{GENDER VIOLENCE IN IPN DEGREE STUDENTS}

\author{
María de la Luz Pirrón Curiel*, Marcela Rojas Ortega*, \\ Lucía Esparza Zamudio*
}

* Maestra. Escuela Superior de Comercio y Administración. Instituto Politécnico Nacional.

** Doctora. Escuela Superior de Comercio y Administración. Instituto Politécnico Nacional. marojas@ipn.mx

*** Licenciada. Escuela Superior de Comercio y Administración. Instituto Politécnico Nacional. lesparza@ipn.mx

Dirección para recibir correspondencia: mpirron@ipn.mx 


\section{VIOLENCIA DE GÉNERO EN ESTUDIANTES DE LICENCIATURA DEL IPN}

\section{RESUMEN}

OBJETIVO: Considerando, que los estudiantes de la Escuela Superior de Comercio, y Administración Unidad Tepepan (ESCA Tepepan), del Instituto Politécnico Nacional (IPN), pueden verse afectados en su formación profesional por la violencia de género, en la cual se realizó una investigación para conocer las experiencias de los estudiantes respecto a este factor. El objetivo del estudio, fue describir la situación actual de los estudiantes, de la ESCA Tepepan, del IPN, respecto a la violencia de género.

MATERIAL Y MÉTODO: Se llevó a cabo, un estudio de campo transversal de tipo descriptivo, en la cual las variables investigadas fueron: violencia física, violencia psicológica, violencia sexual, marginación, y exclusión, o rechazo social. Se empleó el método de interrogatorio, mediante un cuestionario cerrado auto administrado, sobre la violencia de género, ya que se elaboró, y validó específicamente para la investigación, el cual fue aplicado a una muestra no probabilística de los estudiantes del plantel.

RESULTADOS: Se encontró, que la violencia de género se presenta a nivel moderado, y que el tipo de violencia que presenta mayor incidencia, es la violencia psicológica.

CONCLUSIONES: También se incluyen propuestas de acción, para disminuir la violencia de género en el contexto escolar, creando un ambiente de convivencia que propicie, el aprendizaje, y la formación personal, y profesional.

PALABRAS CLAVE: Violencia de género. Nivel universitario. Formación integral. 


\section{VIOLENCIA DE GÉNERO EN ESTUDIANTES DE LICENCIATURA DEL IPN}

\section{ABSTRACT}

OBJECTIVE: Considering that the students of the Higher School of Commerce, and Administration, Tepepan Unit (ESCA Tepepan), of the National Polytechnic Institute (IPN), may be affected in their professional training because of gender violence, an investigation was conducted to know the experiences of the students regarding this factor. The objective of this study was to describe the current situation of the students, of the ESCA Tepepan, of the IPN, regarding the gender violence.

MATERIAL AND METHOD: A descriptive cross-sectional study was carried out, in which the variables investigated were: physical violence, psychological violence, sexual violence, marginalization, and exclusion, or social rejection. The questioning method was used, by means of a self-administered closed questionnaire on gender violence. This questionnaire was developed and validated specifically for the research, and it was applied to a non-probabilistic sample of the students on the campus.

RESULTS: It was found that gender violence occurs at a moderate level, and that the type of violence that has the greatest incidence is psychological violence.

CONCLUSIONS: It also includes proposals for action to reduce gender violence in the school context, creating an atmosphere of coexistence that encourages learning, and the personal, and professional training.

KEY WORDS: Gender violence. University level. Integral formation. 


\section{VIOLENCIA DE GÉNERO EN ESTUDIANTES DE LICENCIATURA DEL IPN}

\section{INTRODUCCIÓN}

La violencia, se define como "La amenaza, o uso de la fuerza física o poder en contra de otras personas, contra uno mismo, contra objetos, o contra un grupo, o comunidad" (Amórtegui, 2005, p. 158). Este fenómeno, ocurre en diferentes contextos, afectando en forma severa el desarrollo del individuo, y su adaptación exitosa al contexto social. En México y otros países, desafortunadamente, la violencia sigue presentándose, y una de sus modalidades más frecuentes es la violencia de género.

Datos de investigaciones a nivel internacional, señalan que la violencia de género, "Es un fenómeno que se da en el ámbito universitario, y como consecuencia, existen numerosas iniciativas, y experiencias en diversas universidades del mundo, que implementan medidas para prevenir situaciones de violencia de género en el contexto universitario" (Aguilar y otros, 2009, pp. 285-286).

En el caso, de la existencia de la violencia de género, las características de los grupos sociales, pueden influir en las formas en que se manifiesta, y se reacciona ante dicho fenómeno. Por ejemplo, en algunos grupos culturales, "Muchos adolescentes resienten ser tocados en cualquier forma. Ocasionalmente los profesores, también pueden hacer enojar a sus alumnos al reaccionar de cualquier manera negativa" (Good \& Brophy, 2001, p. 371). La violencia, puede manifestarse de diferentes maneras, entre las más comunes se encuentran: la violencia física, la violencia psicológica, y la violencia sexual.

Se considera, violencia, o, maltrato físico, a "Cualquier acto que infringe daño no accidental, usando fuerza física, o algún tipo de arma u objeto, que pueda provocar lesiones ya sean internas, externas, o ambas" (UACH, 2015).

La violencia, o maltrato psicológico consiste, en "El uso deliberado del abuso psicológico, incluyendo el maltrato verbal, acoso, aislamiento, y privación de los recursos físicos, financieros, y personales, para controlar, y manipular a la pareja, o, al ambiente más cercano" (EcuRed, 2016).

Se caracteriza por: Desvalorizaciones (críticas y humillaciones permanentes), las posturas, y gestos amenazantes (amenazas de violencia o suicidio), conductas de restricción, (control de las amistades, limitación del dinero o restricción de las salidas de casa), conductas destructivas, 


\section{VIOLENCIA DE GÉNERO EN ESTUDIANTES DE LICENCIATURA DEL IPN}

(referidas a objetos de valor económico o afectivo o al maltrato de animales domésticos) y, por último, culparla de conductas violentas (Tirado \& otros, 2010, p. 189).

La violencia sexual, "Es cualquier acto que degrada, o daña el cuerpo, y/o la sexualidad de la víctima, que por tanto atenta contra su libertad, dignidad, e integridad física" (CONAVIM, 2015, p. 8).

La marginación, exclusión, o rechazo social, es una forma de discriminación, relacionada con el género. Se define, como el "Proceso, por el que una sociedad, o miembros de la misma rechazan, a determinados individuos, desde la simple indiferencia, hasta la represión, y reclusión" (Ayllon \& otros, 2010, p. 47).

Fernández \& Echeburrúa (1997), identificaron algunos aspectos relacionados con la violencia potencial, entre ellos se encuentran: "Ser excesivamente celoso, posesivo, irritarse cuando se le ponen límites, beber alcohol en exceso, culpar a los demás de sus problemas, experimentar cambios repentinos de humor, romper cosas cuando se enojan, y tener baja autoestima" (Tirado \& otros, 2010, pp. 191-192).

Con base en la información bibliográfica, se decidió realizar un estudio sobre la violencia de género, en los estudiantes de la Escuela Superior de Comercio y Administración, Unidad Tepepan (ESCA Tepepan), del Instituto Politécnico Nacional (IPN), para conocer las experiencias de los estudiantes, respecto a este factor, y determinar la situación actual en la comunidad escolar.

Con la finalidad, de contar con información fidedigna, sobre las características de los estudiantes de la ESCA Tepepan, del IPN, en cuanto a la violencia de género, con la finalidad de proponer acciones para disminuirla en el contexto escolar.

\section{PLANTEAMIENTO Y MÉTODO DE INVESTIGACIÓN}

\section{Objetivo general}

Describir, la situación actual de los estudiantes de la ESCA Tepepan, del IPN, respecto a la violencia de género. 


\section{VIOLENCIA DE GÉNERO EN ESTUDIANTES DE LICENCIATURA DEL IPN}

\section{Objetivo específico}

Sugerir acciones, para contribuir al manejo, y disminución de violencia de género en el contexto escolar.

\section{PLANTEAMIENTO DEL PROBLEMA}

La violencia de género, representa un problema social con múltiples implicaciones, actualmente existen estudios sobre este factor en diferentes escenarios, la mayoría caracterizados por enfocarse exclusivamente al sexo femenino. Sin embargo, se consideró importante realizar una investigación, donde se describiera este factor, tanto en hombres, como en mujeres, que forman parte de una misma población escolar.

Debido a lo anterior, se considera fundamental plantear la siguiente pregunta de investigación: ¿Cuál, es la situación actual, en cuanto a la violencia de género de los estudiantes de la ESCA Tepepan?

\section{HIPÓTESIS DE TRABAJO}

La violencia de género, se presenta en los estudiantes de la ESCA Tepepan a nivel moderado.

\section{VARIABLES}

Las variables que se eligieron, para el estudio son: violencia física, violencia psicológica, violencia sexual, y marginación, exclusión, o rechazo social. 


\section{VIOLENCIA DE GÉNERO EN ESTUDIANTES DE LICENCIATURA DEL IPN}

\section{Tabla 1}

Variables

\section{Definición}

Violencia física

"Es cualquier acto, que infringe daño no accidental, usando fuerza física o algún tipo de arma, u objeto que pueda provocar o no lesiones ya sean internas, externas." (UACH, 2015).

Violencia psicológica

"Se designa, como el uso deliberado del abuso psicológico, incluyendo el maltrato verbal, acoso, aislamiento y privación de los recursos físicos, financieros y personales, para controlar, y manipular, a la pareja o al ambiente más cercano. Hay violencia, cuando se ataca la integridad emocional, o espiritual de una persona" (EcuRed, 2016).

Violencia sexual

Es cualquier acto, que degrada o daña el cuerpo y/o la sexualidad de la víctima, y que por tanto atenta contra su libertad, dignidad e integridad física" (CONAVIM, 2015, p. 8).

Marginación, exclusión Proceso, por el que una sociedad o miembros de la misma o rechazo social rechazan a determinados individuos, desde la simple indiferencia hasta la represión y reclusión" (Ayllon \& otros, 2010, p. 47).

Fuente: Elaboración propia (2016). 
VIOLENCIA DE GÉNERO EN ESTUDIANTES DE LICENCIATURA DEL IPN

Tabla 2

Muestra de alumnos participantes en el estudio

\begin{tabular}{ccccc}
\hline $\begin{array}{l}\text { Licenciatura } \\
\text { Género }\end{array}$ & $\begin{array}{c}\text { Lic. Contaduría } \\
\text { Pública }\end{array}$ & $\begin{array}{c}\text { Lic. Relaciones } \\
\text { Comerciales }\end{array}$ & $\begin{array}{c}\text { Lic. Negocios } \\
\text { Internacionales }\end{array}$ & Total \\
\hline Masculino & 218 & 169 & 266 & 653 \\
Femenino & 294 & 197 & 280 & 771 \\
$\begin{array}{c}\text { Total de } \\
\text { participantes }\end{array}$ & 512 & 366 & 546 & 1424 \\
\hline
\end{tabular}

Fuente: Elaboración propia (2016).

La muestra es no probabilística, y quedó integrada por los estudiantes que se encontraban en los salones, de 16 grupos del turno matutino, y 32 del turno vespertino, lo que representa aproximadamente, un $15 \%$ de la población total. Los grupos fueron elegidos, de acuerdo al horario acordado para la aplicación, y a la disposición de los docentes para ceder parte del tiempo de su clase.

\section{INSTRUMENTO DE INVESTIGACIÓN}

Para la realización del estudio, se elaboró un cuestionario cerrado, sobre violencia de género. Dicho cuestionario incluye 45 ítems, los cuales fueron validados por la técnica de jueces, y corresponden a las variables seleccionadas para el estudio.

\section{RESULTADOS}

Para el tratamiento de los datos, se capturó una base de datos en el paquete SPSS Versión 17. Los datos, fueron analizados mediante estadística descriptiva, obteniendo porcentajes por licenciatura, por variable, y por género, para ser posteriormente interpretados cuantitativa, y cualitativamente.

La interpretación de resultados, se llevó a cabo con apoyo de la tabla 3, que fue elaborada de acuerdo a la escala del 1 al 5, usada en el cuestionario de investigación. 


\section{VIOLENCIA DE GÉNERO EN ESTUDIANTES DE LICENCIATURA DEL IPN}

Tabla 3

Criterios de interpretación

\begin{tabular}{lll}
\hline Puntuación & Nivel de Violencia de género \\
\hline 0 a 1 a & Nulo \\
1.01 a 2 & Moderado \\
2.01 a 3 & Considerable \\
3.01 a 4 & Grave \\
4.01 a 5 & Alarmante \\
\hline
\end{tabular}

Fuente: Elaboración propia (2016).

\section{Resultados globales promedio}

Como se observa en la tabla 4, en la mayor parte de los casos, se reportan niveles moderados de violencia de género en la población escolar. Sin embargo, en el grupo de hombres, se reporta violencia psicológica, en un nivel considerable, además tanto en el caso de los estudiantes, esta variable es la que presenta los puntajes más altos.

Lo anterior, permite conocer específicamente como se presenta la violencia, de acuerdo a la experiencia de los estudiantes; es interesante resaltar, que de acuerdo a los resultados obtenidos, los estudiantes masculinos, experimentan más situaciones de violencia que las mujeres en todos los casos.

\section{Tabla 4}

Concentrado de medias globales por variable y género

\begin{tabular}{llll}
\hline Variable & \multicolumn{3}{c}{ Resultado } \\
\hline & Hombres & Mujeres & Promedio \\
Violencia física & 1.83 & 1.65 & 1.74 \\
Violencia psicológica & 2.06 & 1.92 & 1.99 \\
Violencia sexual & 1.72 & 1.68 & 1.70 \\
Marginación, exclusión o rechazo social & 1.82 & 1.66 & 1.74 \\
\hline
\end{tabular}

Fuente: Elaboración propia (2016). 


\section{VIOLENCIA DE GÉNERO EN ESTUDIANTES DE LICENCIATURA DEL IPN}

\section{Niveles de violencia física experimentados por la totalidad de la muestra}

En la figura 1, se observa que la mayor parte de los estudiantes de los dos sexos, reportan haber experimentado un nivel moderado de violencia física, durante los seis meses anteriores a la aplicación del cuestionario. En ambos casos, este nivel es superior al 60\%, aun cuando el porcentaje es mayor en los estudiantes del sexo masculino. La violencia física no se experimenta, en el $25 \%$ de los casos de alumnos, y en el $36 \%$ de alumnas, lo que resulta positivo, aun cuando lo deseable es que no se presente en ninguno de los casos.

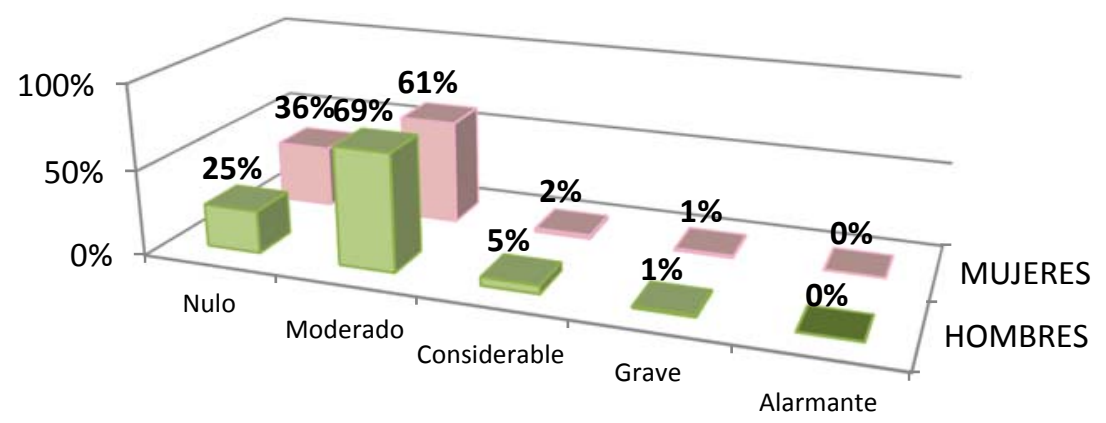

Figura 1. Violencia física experimentada por la totalidad de la muestra.

Fuente: Elaboración propia (2017).

En el nivel denominado considerable, se encuentra un $5 \%$ de los hombres, y un $2 \%$ de las mujeres, lo que representa un riesgo real para la integridad física de los estudiantes. Además, se observa que un $1 \%$ de los estudiantes, tanto hombres como mujeres, han sufrido casos graves de violencia física en épocas recientes. El total de casos de riesgo que requieren atención inmediata, corresponde al 6\% de los hombres, y al 3\% de las mujeres.

Lo anterior, hace indispensable tomar medidas de apoyo inmediatas, para modificar favorablemente, la situación que viven los estudiantes en riesgo, aun cuando el número de estudiantes sea reducido, ya que su integridad física se encuentra en peligro.

\section{Niveles de violencia psicológica de la totalidad de la muestra}

La violencia psicológica, es experimentada en diversos niveles por el 83\% de los hombres, y el $78 \%$ de las mujeres, de acuerdo a la información proporcionada por los encuestados, ya que únicamente el $17 \%$ de los participantes del sexo masculino, y el $22 \%$ del sexo femenino reportan no haber sufrido este tipo de violencia. 
VIOLENCIA DE GÉNERO EN ESTUDIANTES DE LICENCIATURA DEL IPN

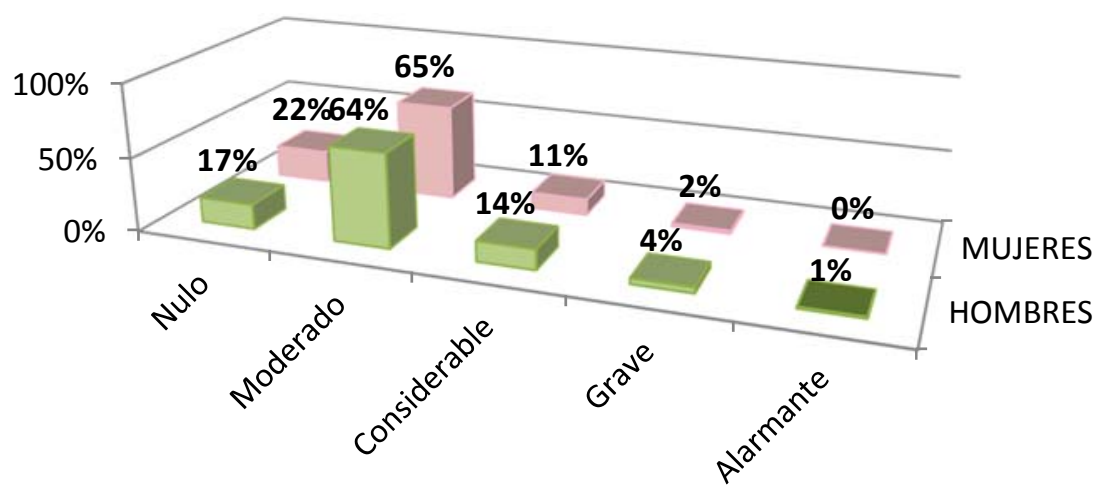

Figura 2. Violencia psicológica experimentada por la totalidad de la muestra.

Fuente: Elaboración propia (2016).

En la figura 2, se observa que un $64 \%$ de los hombres, y un $65 \%$ de las mujeres, perciben que han experimentado violencia psicológica a nivel moderado, lo que no es altamente significativo, aun cuando lo deseable sería que la violencia fuera nula.

Los aspectos que presentan mayor riesgo, en cuanto a la violencia psicológica, son los referidos, en orden ascendente de importancia, como considerables por el 14\% de los hombres, y el $11 \%$ de las mujeres, los considerados graves por el $4 \%$ de los hombres, y el $2 \%$ de las mujeres, y además, existen casos alarmantes reportados por el 1\% de los estudiantes del sexo masculino. Éstos casos incluyen a cerca del $20 \%$ de la población, lo que resulta muy grave, y requiere de atención inmediata, para evitar que se siga afectando negativamente el desarrollo de la personalidad de los estudiantes.

\section{Nivel de violencia sexual por variable y género}

En la figura 3, se observa que el $41 \%$ de los hombres, y el $43 \%$ de las mujeres reportan no haber experimentado ningún tipo de violencia sexual, lo cual es altamente positivo, ya que en ambos casos representan más del $40 \%$ de la población. Por otra parte, el $49 \%$ de alumnos, y el $50 \%$ de las alumnas, reportan haber vivido situaciones con niveles moderados de violencia sexual, lo que indica que aunque no se representan en un punto crítico, estos casos deben ser atendidos.

El 7\% de los hombres, y el $4 \%$ de las mujeres, han experimentado violencia sexual con una frecuencia considerable. El 2\% tanto de hombres, como de mujeres, han sufrido violencia sexual a niveles graves, y un $1 \%$ de los estudiantes, reporta que se presenta a nivel alarmante. 


\section{VIOLENCIA DE GÉNERO EN ESTUDIANTES DE LICENCIATURA DEL IPN}

Estos niveles de frecuencia, representan áreas que requieren atención inmediata, ya que representan un riesgo importante para la población, aun cuando su número sea bajo, ya que pueden causar efectos importantes, y en ocasiones e irreversibles en las personas.

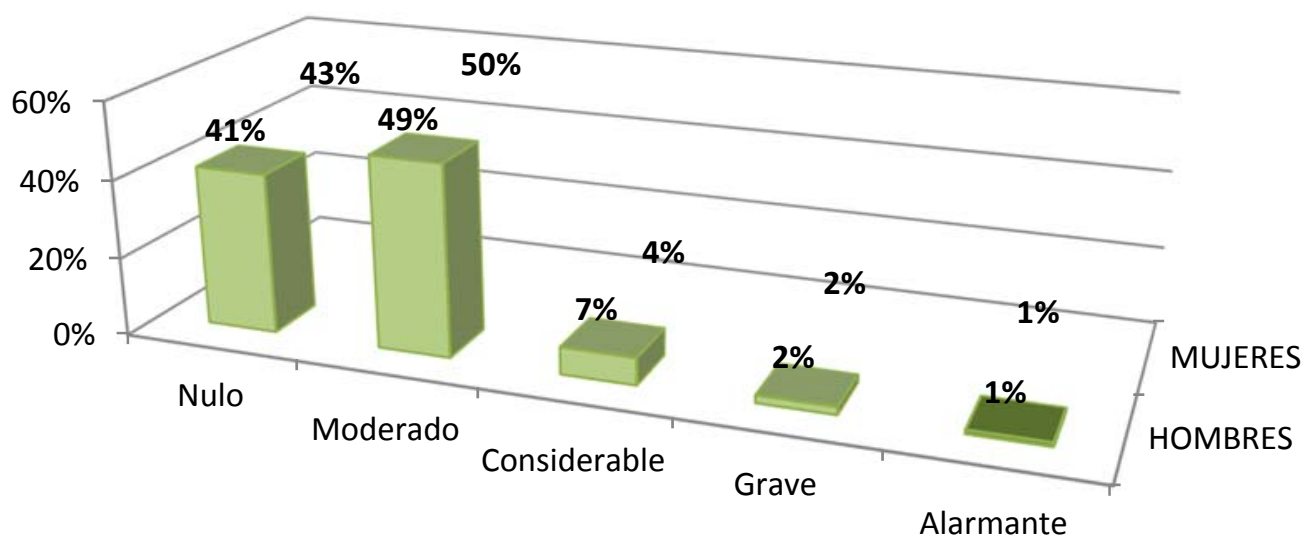

Figura 3. Violencia sexual por género de la totalidad de la muestra.

Fuente: Elaboración propia (2016).

\section{Nivel de marginación, exclusión o rechazo social por variable y género}

De acuerdo, a lo ilustrado en la figura 4, se percibe que el $37 \%$ de los hombres, y el $43 \%$ de las mujeres, refieren que no han experimentado ningún tipo de marginación, exclusión, o rechazo social, en los últimos seis meses, lo que representa un porcentaje alto de estudiantes libres de este tipo de violencia.

En el nivel moderado, se encuentra que el 50\% de la población estudiada, tanto de hombres, como de mujeres, lo que indica un nivel bajo de peligro respecto a esta variable, aun cuando evidentemente si se presenta la marginación, exclusión, o rechazo social, en el contexto escolar, y es un factor que es susceptible de acciones de mejora.

En cuanto a la marginación, y el rechazo social, a niveles que implican mayor riesgo, se encontró que el 9\% de los hombres, y el 6\% de las mujeres sufren algún tipo de marginación, exclusión, o rechazo social, con un nivel considerable de frecuencia, que el $3 \%$ de los hombres, y el $1 \%$ de las mujeres, experimentan niveles graves de este factor, y el $1 \%$ de los hombres lo reporta a nivel alarmante. 
VIOLENCIA DE GÉNERO EN ESTUDIANTES DE LICENCIATURA DEL IPN

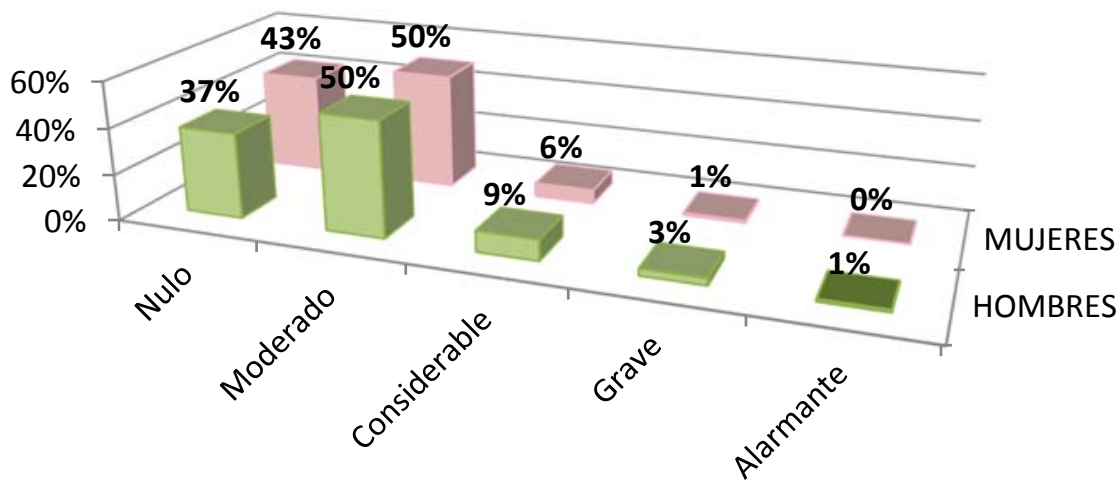

Figura 4. Marginación o rechazo social por variable y género.

Fuente: Elaboración propia (2016).

Si se toma en cuenta, que estos casos representan alrededor del 10\% de la población, puede considerarse que se está ante un problema que requiere pronta atención, ya que puede afectar aspectos psicológico, y sociales, del desarrollo del individuo.

\section{Resultados por licenciatura}

Los resultados promedio, obtenidos por los estudiantes inscritos en las licenciaturas, que se imparten en la ESCA Tepepan, del IPN, se presentan en la tabla 5.

Se observa, que el nivel más alto de violencia reportado en la tabla 5, en promedio, corresponde a considerable, lo cual representa ya un riesgo importante. Los resultados elevados en la variable violencia psicológica, corresponden a alumnos del sexo masculino de las tres licenciaturas, y las alumnas de la licenciatura en Contaduría Pública, así como a los estudiantes hombres de la licenciatura en Relaciones Comerciales, en la variable violencia sexual. 
VIOLENCIA DE GÉNERO EN ESTUDIANTES DE LICENCIATURA DEL IPN

Tabla 5

Resultados hombres y mujeres por licenciatura

\begin{tabular}{|c|c|c|c|c|c|c|}
\hline \multirow{2}{*}{$\begin{array}{l}\text { Licenciatura } \\
\text { Variable }\end{array}$} & \multicolumn{2}{|c|}{ Lic. Contaduría Pública } & \multicolumn{2}{|c|}{ Lic. Relaciones Comerciales } & \multicolumn{2}{|c|}{ Lic. Negocios Internacionales } \\
\hline & Masculino & Femenino & Masculino & Femenino & Masculino & Femenino \\
\hline $\begin{array}{l}\text { Violencia } \\
\text { física }\end{array}$ & 1.86 & 1.59 & 1.80 & 1.64 & 1.80 & 1.59 \\
\hline $\begin{array}{l}\text { Violencia } \\
\text { psicológica }\end{array}$ & 2.01 & 2.01 & 2.40 & 1.89 & 2.02 & 1.84 \\
\hline $\begin{array}{l}\text { Violencia } \\
\text { sexual }\end{array}$ & 1.68 & 1.78 & 2.12 & 1.66 & 1.63 & 1.59 \\
\hline $\begin{array}{l}\text { Marginación, } \\
\text { exclusión o } \\
\text { rechazo } \\
\text { social }\end{array}$ & 1.80 & 1.70 & 1.88 & 1.69 & 1.83 & 1.61 \\
\hline
\end{tabular}

Fuente: Elaboración propia (2016).

Es interesante destacar, el hecho de que los estudiantes hombres, en general, reporten niveles más altos que las mujeres en cuanto a violencia psicológica. Los ítems, que obtuvieron valores más altos de violencia psicológica, en el grupo de hombres, son: engaños, le han realizado bromas hirientes, ser culpados por cosas que no hicieron con chantajes emocionales, en ese orden.

En cuanto, al grupo de mujeres, que estudian la licenciatura de Contaduría Pública, se observa, que presentan los niveles más altos en los mismos ítems que los hombres, lo que muestra que en el caso específico de esta licenciatura, y en la variable mencionada los estudiantes refieren haber experimentado violencia psicológica, en los mismos aspectos, y en niveles bastante similares.

En cuanto, a los estudiantes de la licenciatura en Relaciones Comerciales, las altas puntuaciones obtenidas en la variable violencia sexual, se derivan de las respuestas a los ítems, relacionados con celos de la pareja, ser objeto de manoseos o toqueteos no deseados, y sufrir acoso, en ese orden. 


\section{VIOLENCIA DE GÉNERO EN ESTUDIANTES DE LICENCIATURA DEL IPN}

\section{Acciones propuestas}

A partir, de los resultados obtenidos se advierte la necesidad de realizar acciones, que permitan mejorar la situación actual. Como punto medular, es necesario integrar un plan de atención a los casos de violencia, que sea ampliamente difundido a todos los miembros de la comunidad escolar, para que cualquier integrante de ella pueda tener acceso a recibir orientación, y apoyo.

En el plan de atención, deberán incluirse aspectos preventivos, y remediales, mediante acciones, que puedan realizarse con los recursos con que cuentan la ESCA Tepepan, el Instituto Politécnico Nacional y las instituciones públicas. Lo anterior con el fin de que el desarrollo del plan no dependa de nuevos recursos para su puesta en marcha.

Las acciones que se proponen para dicho plan son:

- Capacitar a autoridades, profesores, y personal de apoyo, y asistencia a la educación, en cuanto al trato respetuoso, digno e igualitario a todos los integrantes de la comunidad escolar.

- Pedir a las autoridades, profesores, personal de apoyo, y asistencia a la educación ser un ejemplo, en cuanto a evitar mostrar cualquier tipo de violencia.

- Propiciar un ambiente de confianza, para que los miembros de la comunidad que requieran orientación, y apoyo, puedan solicitarlo, confiando en que sus casos serán tratados con estricta confidencialidad.

- Proporcionar, atención psicológica específica, para casos de violencia de género en el área de Orientación Educativa del plantel.

- Brindar, cursos de desarrollo personal, manejo de la agresión, comunicación asertiva, manejo de conflictos, y relaciones humanas.

- Elaborar, y distribuir carteles, trípticos, y folletos con información sobre cómo identificar, y combatir la violencia de género.

- Brindar información, a través de la red de género de la ESCA Tepepan, y, del IPN.

- Implementar eventos que refuercen la formación en aspectos de género como: conferencias, mesas redondas, debates, análisis de películas, y textos. 


\section{VIOLENCIA DE GÉNERO EN ESTUDIANTES DE LICENCIATURA DEL IPN}

\section{CONCLUSIONES}

A partir de la investigación realizada, se acepta la hipótesis de trabajo, debido a que se concluye que la violencia de género es experimentada, en promedio, a nivel moderado, por los estudiantes de la ESCA Tepepan de las tres licenciaturas que se imparten en el plantel, (Contaduría Pública, Relaciones Comerciales, y Negocios Internacionales).

Al analizar las variables, los resultados indican que aunque en general no existen problemas extremos, deben tomarse medidas para disminuir la violencia de género, debido a los efectos que tiene en la formación integral de los estudiantes.

En el estudio se observa, que los estudiantes del sexo masculino, reportan situaciones de mayor violencia de género, que en las mujeres en todas las variables, sobresaliendo en puntuación la variable violencia psicológica, (que corresponde a un nivel considerable), seguida por la violencia física, la marginación, o, exclusión, y la violencia sexual, mismas que presentan un nivel moderado. Por otra parte, las estudiantes experimentan mayores niveles en la variable violencia psicológica, al igual que el grupo de hombres, seguida por la variable violencia sexual, la variable marginación, exclusión, o, rechazo social, y con la frecuencia menor se encuentra la variable violencia física.

En cuanto a tendencia, se observa que la violencia sexual, se presenta en segundo lugar de frecuencia, en el grupo de mujeres, mientras que en el grupo de hombres, ocupa el nivel más bajo. Un fenómeno similar ocurre en el caso de los hombres, ya que reportan en segundo lugar la violencia física, y las mujeres en cuarto.

El impacto de este estudio, radica en que proporciona información reciente, y objetiva de los estudiantes en un contexto específico, lo que permite contar con datos que puedan servir como base, para establecer planes de acción para atender la violencia de género, y continuar realizando investigación en este campo.

Se propone crear un plan de atención para la violencia de género con un enfoque preventivo, y remedial, incluyendo acciones que puedan realizarse sin un gasto excesivo, empleando los recursos humanos, y materiales con que ya se cuenta, tanto en el Instituto Politécnico Nacional, como en las instituciones públicas a las que pueden canalizarse los casos más graves. Además, se sugiere incluir en dicho plan aspectos como: capacitación de autoridades, profesores, y personal de apoyo, colaboración de Orientación Educativa, implementación, e 


\section{VIOLENCIA DE GÉNERO EN ESTUDIANTES DE LICENCIATURA DEL IPN}

impartición de diversos cursos, difusión de información por diferentes medios, y la realización de eventos de sensibilización.

\section{REFERENCIAS BIBLIOGRÁFICAS}

Aguilar (marzo 2009). Violencia de género en el ámbito universitario. Medidas para su superación. Revista interuniversitaria de pedagogía social (SIPS), 16, Competencias, y Profesionalización, Tercera época. Recuperado de: https://recyt.fecyt.es/index.php/PSRI/article/view/36950

Amórtegui-Osorio. (Junio 2005). Violencia en el Ámbito Universitario el caso de la Universidad Nacional de Colombia. Revista Salud Pública 7(2). Recuperado de: https://pdfs.semanticscholar.org/738d/6a1c94ee5e23dec0a429d8c4740e25e54e08.p df

Ayllon \& otros. (2010). Diversidad cultural: ciudadanía, política, y derecho. Biblioteca Virtual de Derecho, Economía, y Ciencias Sociales. Recuperado de: http://www.eumed.net/libros

CONAVIM. (2015). Estudio Nacional sobre las fuentes, orígenes, y factores que producen, $y$ reproducen la violencia hacia las mujeres. I (I). Recuperado de: http://www.conavim.gob.mx/work/models/CONAVIM/Resource/103/1/images/Estudio sNacionalesTomolVolumenl.pdf

EcuRed. (2016). Enciclopedia cubana. Recuperado de: https://www.ecured.cu/Violencia_psicol\%C3\%B3gica

Good y Brophy. (2001). Psicología Educativa Contemporánea. México: McGraw Hill.

Tirado (2010). Psicología Educativa. México: McGraw Hill. 